\title{
A New Section in MASA: Guide Handbook of Statistical Techniques (GHOST)
}

Researchers and practitioners often need to get accessible information without having to look through tons of content to find it. For this aim, we in MASA are organizing a new section of the Guide Handbook of Statistical Techniques (GHOST).

Such a reference guide is a documentation that provides a description on various models, methods, methodologies, and their applications within a chosen topic. A work in this section would present a brief review based on a set of references detailed at the level of the abstracts of the previously issued papers, or in a slightly extended form.

An author of such a collection of information can mostly describe his/her own works issued in professional sources including MASA, supplying the references to them, possibly with links to the related websites and repositoria of the papers. If available due to limitations on the permitted author's copies, the authors could be able to supply the interested readers with the required works upon request.

This new section as a compendium of information and source of available papers, would raise further activity around the journal and attract more authors and readers to MASA. 